\title{
Mechanisms of Potentiation of Mossy Fiber EPSCs in the Cerebellar Nuclei by Coincident Synaptic Excitation and Inhibition
}

\author{
Jason R. Pugh ${ }^{1}$ and Indira M. Raman ${ }^{1,2}$ \\ ${ }^{1}$ Interdepartmental Neuroscience Program and ${ }^{2}$ Department of Neurobiology and Physiology, Northwestern University, Evanston, Illinois 60208
}

\begin{abstract}
Neurons of the cerebellar nuclei receive synaptic excitation from cerebellar mossy fibers. Unlike in many principal neurons, coincident presynaptic activity and postsynaptic depolarization do not generate long-term potentiation at these synapses. Instead, EPSCs are potentiated by high-frequency trains of presynaptic activity applied with postsynaptic hyperpolarization, in patterns resembling mossyfiber-mediated excitation and Purkinje-cell-mediated inhibition that are predicted to occur during delay eyelid conditioning. Here, we have used electrophysiology and Ca imaging to test how synaptic excitation and inhibition interact to generate long-lasting synaptic plasticity in nuclear cells in cerebellar slices. We find that the extent of plasticity varies with the relative timing of synaptic excitation and hyperpolarization. Potentiation is most effective when synaptic stimuli precede the postinhibitory rebound by $\sim 400 \mathrm{~ms}$, whereas with longer intervals, or with a reverse sequence, EPSCs tend to depress. When basal intracellular Ca is raised by spontaneous firing or reduced by voltage clamping at subthreshold potentials, potentiation is induced as long as the synaptic-rebound temporal sequence is maintained, suggesting that plasticity does not require Ca levels to exceed a threshold or attain a specific concentration. Although rebound and spike-dependent Ca influx are global, potentiation is synapse specific, and is disrupted by inhibitors of calcineurin or Ca-calmodulindependent protein kinase II, but not PKC. When IPSPs replace the hyperpolarizing step in the induction protocol, potentiation proceeds normally. These results lead us to propose that synaptic and inhibitory/rebound stimuli initiate separate processes, with local NMDA receptor-mediated Ca influx "priming" synapses, and Ca changes from the inhibition and rebound "triggering" potentiation at recently activated synapses.
\end{abstract}

Key words: deep cerebellar nuclei; interpositus; Purkinje cell; synaptic plasticity; eye blink; long-term potentiation; coincidence detection; non-Hebbian

\section{Introduction}

Neurons of the cerebellar nuclei, which receive excitatory synaptic input primarily from mossy fibers and inhibitory synaptic input from Purkinje cells, form the major output of the cerebellum. Consequently, most cerebellar learning must manifest itself as changes in the activity of cerebellar nuclear neurons. For instance, in delay eyelid conditioning, well timed increases in nuclear neuron activity allow trained animals to close their eyelids in response to initially neutral "conditioned" stimuli (carried by mossy fibers) that predict reflex-eliciting "unconditioned" stimuli (carried by climbing fibers), such as an air puff to the eye (McCormick and Thompson, 1984; Mauk et al., 1986; Steinmetz et al., 1989; Hesslow et al., 1999). Behavioral studies demonstrate that this learning process occurs in two stages, with synaptic

Received May 5, 2008; revised Aug. 1, 2008; accepted Sept. 2, 2008.

This work was supported by National Institutes of Health Grant NS39395 (I.M.R.) and National Research Service Award F31NS055542 (J.R.P.). We are especially grateful to Dr. Abigail Person for contributing the control experiment demonstrating potentiation in the presence of the calcium indicator. We also thank Teresa Aman, Nan Zheng, Jason Bant, Mark Benton, and Abigail Person for helpful discussion.

Correspondence should be addressed to Indira M. Raman, Department of Neurobiology and Physiology, 2205 Tech Drive, Northwestern University, Evanston, IL 60208. E-mail: i-raman@northwestern.edu.

DOI:10.1523/JNEUROSCI.2061-08.2008

Copyright $\odot 2008$ Society for Neuroscience $\quad$ 0270-6474/08/2810549-12\$15.00/0 and/or excitability changes occurring first in Purkinje cells, and second in nuclear cells (Hesslow, 1994; Garcia and Mauk, 1998; Ohyama and Mauk, 2001; Ohyama et al., 2006). Moreover, modeling studies predict that, during acquisition of conditioned responses, Purkinje neurons generate instructive signals that regulate the strength of mossy fiber synapses onto nuclear cells, ultimately changing nuclear cell output to produce adaptive responses (Miles and Lisberger, 1981; Medina and Mauk, 1999).

This prediction, which requires nuclear cells to integrate inhibitory and excitatory stimuli, is supported by studies of synaptic plasticity in nuclear neurons in cerebellar slices (Pugh and Raman, 2006). This work indicates that EPSCs from mossy fiber afferents are potentiated by high-frequency trains of synaptic excitation only when they are combined with hyperpolarization of the postsynaptic nuclear neuron. Potentiation requires a Ca rise in the postsynaptic cell, activation of NMDA receptors, and concerted activation of $\mathrm{Ca}$ channels after the applied hyperpolarization, which produces the "rebound" depolarization (or rebound current) that typifies many nuclear neurons (Llinás and Mühlethaler, 1988; Aizenman and Linden, 1999). Although nuclear neurons generally fire spontaneously (Jahnsen, 1986a), potentiation does not require spiking and can occur under voltage clamp. 
The induction protocols in our previous study mimicked the predicted time course of excitation and inhibition during delay eyelid conditioning. The conditions were not maximally physiological, however, in that spontaneous firing was prevented, current injection replaced synaptic inhibition, and, in some experiments, cells were voltage clamped. Nevertheless, the fact that these protocols induced plasticity at synapses resistant to Hebbian conditioning (Aizenman and Linden 2000) suggests that they indeed converged on a physiological mechanism. This idea raises the question of how the key elements of the initial protocols might occur under physiological conditions to generate potentiation. Here, we have combined electrophysiology, Ca imaging, and pharmacology to assess the interactions between signals produced by synaptic excitation and by the hyperpolarization and subsequent rebound. The results suggest that potentiation of EPSCs can occur against a wide range of baseline Ca levels, providing evidence against an induction rule requiring dendritic $\mathrm{Ca}$ to reach a threshold concentration. The data instead lead to the hypothesis that excitatory synaptic stimulation provides a cellular signal that "primes" specific synapses, whereas the inhibition/ rebound yields a distinct signal that "triggers" potentiation at primed synapses.

\section{Materials and Methods}

Preparation of cerebellar slices. In accordance with institutional guidelines, cerebellar slices containing the lateral and interpositus nuclei were prepared from 13 to 16-d-old C57BL/6 mice (Charles River Laboratories) as previously described (Telgkamp and Raman, 2002). Mice were deeply anesthetized with halothane and perfused with ice-cold $\left(4^{\circ} \mathrm{C}\right)$ artificial CSF (ACSF) (in mм: $123.75 \mathrm{NaCl}, 3.5 \mathrm{KCl}, 26 \mathrm{NaHCO}_{3}, 1.25$ $\mathrm{NaH}_{2} \mathrm{PO}_{4}, 1.5 \mathrm{CaCl}_{2}, 1 \mathrm{MgCl}_{2}$, and 10 glucose, $\mathrm{pH}$ 7.4). After decapitation, the cerebellum was removed and placed in ice-cold ACSF. Parasagittal slices were cut using a Vibratome (model VT 100S; Leica) and incubated in warm $\left(35^{\circ} \mathrm{C}\right)$ oxygenated $\left(95 \% \mathrm{O}_{2} / 5 \% \mathrm{CO}_{2}\right)$ ACSF for at least $1 \mathrm{~h}$ before recording.

Electrophysiological recording. Cerebellar slices were placed in the recording chamber and bathed in warmed $\left(33-35^{\circ} \mathrm{C}\right)$ oxygenated ACSF containing the $\mathrm{GABA}_{\mathrm{A}}$ receptor antagonist SR95531 (10 $\left.\mu \mathrm{M}\right)$ (Tocris) unless otherwise noted. $\mathrm{GABA}_{\mathrm{B}}$ receptor-mediated currents do not contribute significantly to postsynaptic currents in nuclear neurons (Pugh and Raman, 2005), even with high-frequency stimulation of Purkinje afferents (Morishita and Sastry, 1995; Mouginot and Gähwiler, 1995; Telgkamp et al., 2004). Large projection neurons of the cerebellar nuclei (soma 15-25 $\mu \mathrm{m}$ ) were identified visually with infrared differential interference contrast microscopy. Patch pipettes were pulled from borosilicate glass to a resistance of 3-4.5 M $\Omega$ on a Sutter P97 puller (Sutter). Pipettes were filled with intracellular solution containing the following (in mM): $130 \mathrm{~K}$-gluconate, $2 \mathrm{Na}$-gluconate, $6 \mathrm{NaCl}, 2 \mathrm{MgCl}_{2}$, 14 Triscreatine phosphate, 4 MgATP, 0.3 Tris-GTP, 1 EGTA, 10 HEPES, and 10 sucrose, buffered to $\mathrm{pH} 7.4$ with $\mathrm{KOH}$. Where indicated, calcineurin autoinhibitory fragment (EMD Chemicals), tacrolimus (FK-506; Sigma), Ca-calmodulin-dependent protein kinase II (CaMKII) fragment 290-309 (Sigma), or PKC inhibitor peptide 19-31 (EMD Chemicals) were added to the intracellular solution, and KN62 (Tocris) and FK-506 were added to the bath. Recordings were made with an Axopatch 200B amplifier (Axon Instruments). Data were filtered at $2 \mathrm{kHz}$ and sampled at $50 \mathrm{kHz}$ with pClamp acquisition software (Axon Instruments).

EPSCs were evoked by stimulating the white matter surrounding the cerebellar nuclei with $45 \mu$ s voltage pulses delivered through a concentric bipolar electrode (FHC). The stimulation is likely to activate primarily mossy fibers, which form the bulk of the excitatory synapses onto the proximal dendrites of nuclear cells, although inferior olivary collaterals also form synapses onto the more distal dendrites (Chan-Palay, 1977; Zhang and Linden, 2006; Pugh and Raman, 2006). Baseline EPSC amplitudes were measured at $-65 \mathrm{mV}$ by stimulating pairs of EPSCs separated by $50 \mathrm{~ms}$ once per $15 \mathrm{~s}$ for $5-10 \mathrm{~min}$. Cells with unstable baselines were discarded. After recording baseline EPSCs, the amplifier was switched to current clamp mode (except as noted) to allow the cell to fire normally during the application of the induction protocol. Holding current was applied as indicated. Induction protocols consisted of a $250 \mathrm{~ms}, 133 \mathrm{~Hz}$ train of excitatory synaptic stimuli paired with either a $150 \mathrm{~ms}$ hyperpolarizing current injection or, as noted, a 100-Hz train of inhibitory synaptic stimuli. This pattern of stimulation was repeated 30 times at 5-s intervals. After induction, EPSC amplitudes were again measured at -65 $\mathrm{mV}$ by stimulating pairs of EPSCs once per $15 \mathrm{~s}$ for $20-30 \mathrm{~min}$. For all cells, the percentage change in EPSC amplitude was calculated by comparing the mean baseline amplitude to the mean of all EPSCs recorded after stabilization of the postinduction amplitude.

In experiments in which stimulation of IPSPs replaced hyperpolarizing current injections, SR95531 was omitted from the bathing solution and two stimulating electrodes were inserted into the white matter surrounding the cerebellar nuclei. One electrode was placed near the base of the cerebellar nuclei where mossy fibers entering the cerebellum are abundant and Purkinje axons are relatively sparse. The majority of responses evoked by stimulating this area are exclusively excitatory. This general observation was confirmed at the beginning of each experiment by measuring the evoked current at $-35 \mathrm{mV}$, a voltage at which inhibitory currents, if they are present, are expected to dominate. If outward current was detected at $-35 \mathrm{mV}$, the electrode was repositioned until the outward current was eliminated, or the cell was not analyzed further. EPSCs evoked by this electrode were used to monitor changes in synaptic strength before and after the induction protocol. A second stimulating electrode, placed in or near the corticonuclear tract, was used to elicit IPSPs during the induction protocol. This electrode generally evoked a mix of excitatory and inhibitory responses, but IPSPs dominated such that the net effect was to silence firing in the nuclear neuron.

Ca imaging. EGTA was omitted from the intracellular solution and 200 $\mu \mathrm{M}$ Oregon Green BAPTA 488-1 (Invitrogen) was applied through the patch pipette. Cells were imaged with an Andor EMCCD camera (model DV885KCS-VP, Andor Technology) and TILLvisION software (Till Photonics). Images were captured at $12.5-20 \mathrm{~Hz}$. Basal fluorescence was sufficient to allow visualization of dendrites to $\sim 100 \mu \mathrm{m}$ from the soma, which is $\sim 60-100 \%$ of the length of dendrites of these neurons (Uusisaari et al., 2007). Images were collected only from short stretches $(20-50$ $\mu \mathrm{m})$ of dendrite, because most dendrites did not remain in the focal plane for longer distances.

Excitatory synapses were identified in voltage clamp by evoking 100 $\mathrm{Hz}$ trains of 40 EPSCs while imaging a stretch of dendrite and then searching (on-line) for regions along the dendrite that displayed a stimulus-dependent increase in the fluorescent signal. These regions could generally be isolated to a $2-3 \mu \mathrm{m}$ length of the dendrite. After putative synapses were identified, fluorescence signals were recorded from that region during application of induction protocols judged as "effective" and "ineffective" in potentiation experiments. Photobleaching was minimized by limiting the exposure to short (10-20 ms) durations. After subtraction of background fluorescence, the signal was calculated as the change in fluorescence over baseline $(\Delta F / F)$. Dye saturation during induction protocols was assessed by switching the amplifier to current-clamp mode and measuring the fluorescence signal evoked when cells held at hyperpolarized potentials were made to produce bursts of action potentials by injection of depolarizing current steps. These fluorescence changes were generally an order of magnitude larger than changes produced by the induction protocols in voltage clamp, suggesting that the signals of interest were not near saturation.

Data were analyzed with IGOR software (Wavemetrics) and are presented as mean \pm SE. Statistical significance was assessed with Student's two-tailed paired $t$ tests, except as noted. Stimulus artifacts have been digitally removed or reduced. All reported voltages are corrected for the junction potential of $5 \mathrm{mV}$.

\section{Results}

\section{Plasticity depends on the relative timing of excitation and hyperpolarization}

Our previous work (Pugh and Raman, 2006) indicated that mossy-fiber-mediated EPSCs in cerebellar nuclear neurons were potentiated by repeated applications of a train of EPSCs followed 



Figure 1. Plasticity of EPSCS in the cerebellar nuclei depends on the relative timing of synaptic excitation and the rebound. $\boldsymbol{A}$, Left, Representative responses evoked by three different induction protocols. Cells were held near $-65 \mathrm{mV}$. Protocols consisted of a $250 \mathrm{~ms}, 133 \mathrm{~Hz}$ stimulation of excitatory afferents and a $150 \mathrm{~ms}$ current injection to hyperpolarize the membrane to approximately $-85 \mathrm{mV}$. Bars above each trace indicate the time and duration of synaptic excitation. Right, Representative EPSCs before and after the induction protocols at left. $\boldsymbol{B}$, Top, Mean change in EPSC amplitude before and after induction protocols with the onset of synaptic stimulation occurring $400 \mathrm{~ms}$ before the rebound (closed black circles), 600 ms before the rebound (open, gray-lined triangles), and $150 \mathrm{~ms}$ after the rebound (open, gray-lined squares). Induction protocols in this and similar graphs were applied at time 0 . Bottom, Mean paired-pulse ratio ( 50 ms interval) before and after induction of potentiation with the topmost protocol shown in $\boldsymbol{A}$. C, Percentage change in EPSC amplitudes versus the onset of synaptic stimulation relative to the offset of the hyperpolarization (onset of rebound). Circles indicate mean and SE. Bars indicate the time and duration of synaptic stimulation. Solid black and gray bars correspond to data in $\boldsymbol{A}$ and $\boldsymbol{B}$. Dotted bars represent data from Pugh and Raman (2006). by a post-hyperpolarization rebound depolarization (or current). To investigate the temporal relationship between excitation and hyperpolarization in potentiation of mossy fiber EPSCs, we developed a series of induction protocols in which the onset of synaptic excitation was varied relative to postsynaptic hyperpolarization. Each induction protocol consisted of a $250 \mathrm{~ms}, 133 \mathrm{~Hz}$ stimulation of excitatory afferents (the "synaptic" stimulus) and a $150 \mathrm{~ms}$ hyperpolarization to approximately $-85 \mathrm{mV}$, which was followed by spontaneous, postinhibitory action potentials (the "rebound"). The onset time of the synaptic stimuli was varied from $600 \mathrm{~ms}$ before the rebound to $150 \mathrm{~ms}$ after the rebound. Enough holding current was applied to keep the membrane potential near $-65 \mathrm{mV}$ before stimulation, and was maintained throughout induction. When the synaptic stimulus began $400 \mathrm{~ms}$ before the rebound, potentiation was both robust, increasing EPSC amplitudes by $39 \pm 7 \%(N=9, p<0.001)$, and reliable, potentiating EPSCs by a criterion level of $15 \%$ in eight of nine cells (Fig. $1 A, B$, top). As reported previously (Pugh and Raman, 2006), after application of the induction protocol, EPSCs in most cells were initially depressed. Over the next few minutes, their amplitudes increased, reaching a stable level of potentiation after $\sim 10$ min. Potentiation did not change either the variance of EPSC amplitudes $\left(2328 \pm 871 \mathrm{pA}^{2}\right.$ vs $\left.3327 \pm 962 \mathrm{pA}^{2}, p=0.1\right)$ or the paired-pulse ratio of EPSCs ( $50 \mathrm{~ms}$ interval, $0.82 \pm 0.05 \mathrm{vs}$ $0.79 \pm 0.05, p=0.5$ ) (Fig. $1 B$, bottom), consistent with a postsynaptic locus of plasticity.

When the synaptic stimulus began $>400 \mathrm{~ms}$ before the rebound, however, EPSCs failed to potentiate, instead depressing by $11 \pm 5 \%(N=7, p=0.09)$ (Fig. $1 A$, middle, $B)$. Similarly, EPSCs tended to depress when synaptic stimuli followed the rebound by $150 \mathrm{~ms}(-11 \pm 5 \%, p=0.06, N=8)$ (Fig. $1 \mathrm{~A}$, bottom, $B)$, consistent with work showing that pairing synaptic excitation and postsynaptic depolarization (without hyperpolarization) induces depression (Zhang and Linden, 2006). These results demonstrate that potentiation depends not only on the sequence of stimuli but also on the interval between excitation and inhibition, suggesting that the synaptic stimulus produces a transient signal that lasts a few hundred milliseconds (Fig. 1C).

Our previous work indicates that an increase in postsynaptic $\mathrm{Ca}$ is required for plasticity, because potentiation is blocked by NMDA receptor antagonists, by $10 \mathrm{~mm}$ BAPTA (1,2-bis $(O$ aminophenoxy)ethane- $N, N, N^{\prime}, N^{\prime}$-tetraacetic acid) in the cerebellar nuclear cell, and by voltage ramp protocols that prevent concerted activation of voltage-gated Ca channels after the period of hyperpolarization (Pugh and Raman, 2006). The simplest interpretation of these data is that the synaptic stimulus activates $\mathrm{Ca}$ influx through NMDA receptors and the rebound, through voltage-gated Ca channels, both of which are necessary for plasticity. It is also possible that the hyperpolarization itself plays a specific signaling role, although neither our previous studies nor the data of Figure 1 provide evidence for or against this possibility. The additional requirement for a precise temporal relationship between the synaptic and rebound stimuli raises multiple possibilities for how the signals that they generate might interact to induce plasticity. Potentiation may follow a "threshold rule," in which two stimuli generate intracellular Ca signals that combine to produce a $\mathrm{Ca}$ increase large enough to induce potentiation, possibly incorporating Ca-induced Ca release or a Ca spike (Wang et al., 2000; Brenowitz and Regehr, 2005; Egger et al., 2005). A corollary of this possibility is that potentiation may follow an "inverse-threshold rule," such that moderate Ca rises induce potentiation, but large Ca rises promote depression, as at parallel fiber synapses onto Purkinje cells (Coesmans et al., 2004; 
Belmeguenai and Hansel, 2005). Alternatively, potentiation may follow a "priming rule," in which synaptic stimuli initiate a signaling cascade that provides a short-lived label of activated synapses, and the rebound initiates a separate cascade that triggers potentiation only at "primed" synapses, loosely analogous to synaptic tagging (Frey and Morris, 1997; Martin et al., 1997).

Regarding any type of threshold rule, a potentially complicating factor is that cerebellar nuclear cells fire spontaneously at 20-30 spikes/s (Thach, 1968; Jahnsen, 1986a; Raman et al., 2000; Telgkamp and Raman, 2002), which may generate a considerable $\mathrm{Ca}$ influx through voltage-gated $\mathrm{Ca}$ channels. Imaging studies have demonstrated the presence of voltage-gated Ca channels on nuclear cell dendrites (Muri and Knöpfel, 1994; Gauck et al., 2001) and, because most excitatory synapses are on dendritic shafts rather than spines of nuclear cells (Chan-Palay, 1977), synaptic sites may not be fully isolated from Ca influx associated with spiking. In all induction protocols that we have tested, however, spontaneous firing was prevented by tonic current injection or by voltage clamp (Pugh and Raman, 2006), which is likely to minimize basal Ca levels. If potentiation is sensitive to absolute levels of $\mathrm{Ca}$, an increase in basal $\mathrm{Ca}$ during spontaneous firing might modify the robustness or reliability of potentiation in response to protocols previously characterized as effective.

Therefore, to test whether ongoing firing influences the extent or sign of potentiation, we applied the most effective induction protocol of Figure 1, i.e., with the synaptic stimulus beginning $400 \mathrm{~ms}$ before the rebound, to current-clamped neurons that were allowed to fire spontaneously at $20-30 \mathrm{~Hz}$ between each of the 30 repetitions of the protocol. Under these conditions, EPSCs were potentiated in all six cells tested, increasing by $60 \pm 13 \%$ $(p=0.01)$ (Fig. $2 A$, top, $B)$. In general, inducing potentiation against a background of spontaneous firing appeared more reliable $(100 \%)$ and more robust ( $>30 \%$ amplitude increase in all cells) than when cells were held silent between each sweep. When the synaptic stimulation began $150 \mathrm{~ms}$ after the rebound, however, EPSCs did not potentiate $(-2 \pm 2 \%$ relative to control, $N=$ $5, p=0.8$ ), despite the presumed elevated level of baseline Ca (Fig. $2 A$, middle, $B$ ).

As mentioned, synaptic stimuli before a posthyperpolarization rebound current can potentiate EPSCs even when action potential firing is prevented by voltage clamp (Pugh and Raman, 2006) (data replotted in Fig. 2C). To test the sensitivity of the voltage-clamp induction protocol to the temporal sequence of the synaptic and rebound stimuli, we repeated the experiment with synaptic stimulation applied after the rebound (Fig. $2 A$, bottom, $B$ ). Consistent with the current-clamp experiments, this pattern of stimulation did not potentiate EPSCs $(-24 \pm 25 \%$ relative to control; $N=4, p=0.63)$. Figure $2 C$ summarizes the extent of potentiation measured in current clamp with spontaneous firing permitted, in current clamp with spontaneous firing prevented, and in voltage clamp with no firing at all. Although the amount of potentiation appears greater in protocols with more spiking, the primary observation is that potentiation does not depend on action potential firing. Instead, under all conditions, potentiation is predicted by the order of the synaptic and rebound stimuli, suggesting that the temporal pattern of stimulation is of greater importance than absolute $\mathrm{Ca}$ levels.

Dendritic Ca signals evoked by synaptic and rebound stimuli To begin to investigate this idea directly, we measured relative changes in Ca-dependent fluorescence signals $(\Delta F / F$, referred to as Ca signals) evoked by synaptic and rebound stimuli in cerebel-


C



Figure 2. Potentiation is independent of the amount of spiking during induction. $A$, Representative responses of nuclear neurons to induction protocols consisting of synaptic stimuli (bars) before (top) or after (middle) the hyperpolarizing current injection and subsequent rebound, applied against a background of spontaneous firing, or synaptic stimuli applied after the rebound in voltage clamp (bottom). The voltage steps, from -65 to -80 to $-55 \mathrm{mV}$, are shown above the current trace. $\boldsymbol{B}$, Percentage change in EPSC amplitudes before and after induction protocols shown in $A$. VC, voltage clamp; CC, current clamp; syn, synaptic stimuli; rbd, rebound stimuli. C, Mean change in EPSC amplitudes after induction protocols consisting of synaptic stimuli either before or after the rebound. Induction protocols were given in current clamp with and without spontaneous firing and in voltage clamp holding the cell at $-65 \mathrm{mV}$ between each sweep. The data point for VC, syn before rbd, is from Pugh and Raman (2006). 
A
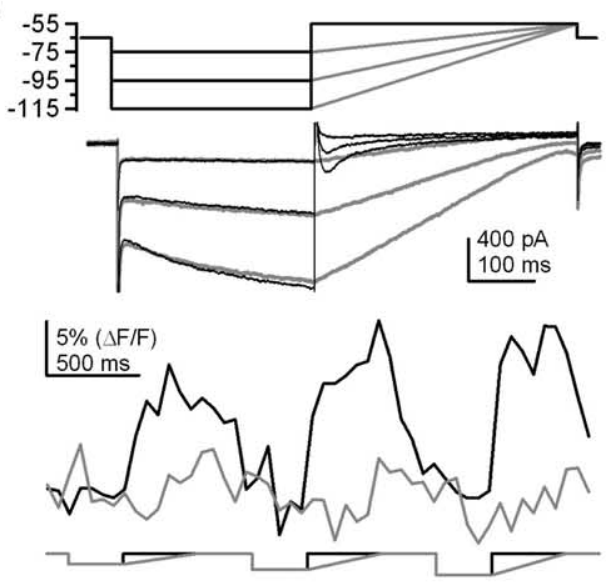

B

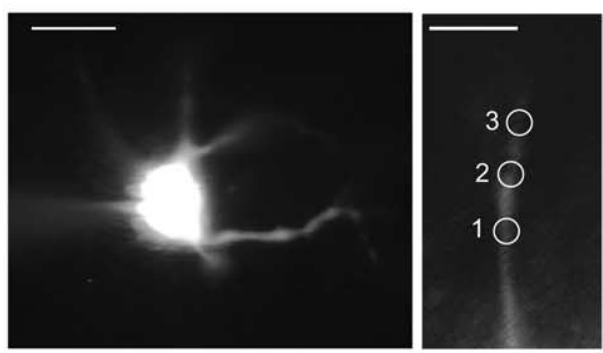

C

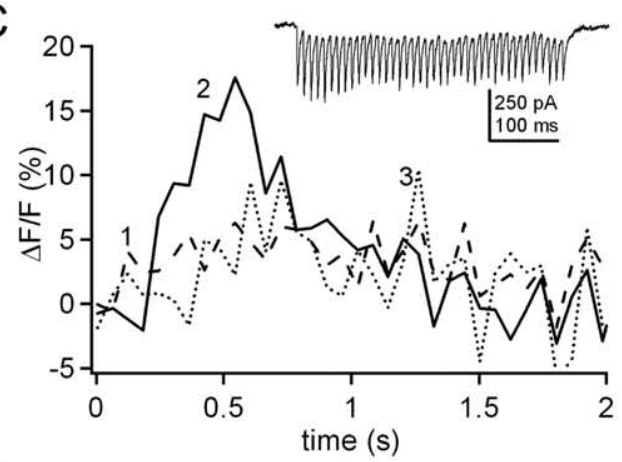

D



Figure 3. Ca signals evoked in nuclear cells by rebound and synaptic stimuli.A, Top, Voltage-clamp protocol and overlaid currents evoked by $300 \mathrm{~ms}$ steps from $-65 \mathrm{mV}$ to $-75,-95$, and $-115 \mathrm{mV}$, followed by either a step (black) or a $400 \mathrm{~ms}$ ramp (gray) to $-55 \mathrm{mV}$. Bottom, Ca signals evoked in a dendrite during the step and ramp voltage-clamp protocols shown above (same cell). Responses to the threestep protocols are concatenated, with conditioning at $-75 \mathrm{mV}$ shown first. Voltage protocol is shown beneath the trace. $\boldsymbol{B}$, Image of a nuclear neuron (left) and of a single dendrite (right) filled with Oregon Green BAPTA $488-1$. Scale bars, $25 \mu \mathrm{m}$ (left) and $5 \mu \mathrm{m}$ (right). C, Ca signals evoked by synaptic stimulation in a nuclear cell held at $-65 \mathrm{mV}$. Numbered traces indicate Ca signals from sites corresponding to numbered circles in $\boldsymbol{B}$. Inset, EPSCs simultaneously recorded in the nuclear cell. $\boldsymbol{D}$, Mean Ca signals measured along the length of dendrites during synaptic stimulation (syn stim.) of 40 EPSCs at $100 \mathrm{~Hz}$ (closed black squares), rebound stimulation (rebound stim.) of steps from -75 to $-55 \mathrm{mV}$ (open triangles), or no stimulation (no stim.; closed gray circles). For each cell, a distance of zero was defined as the site producing the maximal synaptically evoked Ca signal, and distances were measured relative (rel.) to that location. lar nuclear neurons. We first tested whether both synaptic and rebound stimuli-evoked $\mathrm{Ca}$ transients could be detected under our recording conditions. The heavy myelination of this region of the cerebellum presents a challenge in the detection of dendritic Ca signals. We therefore used the relatively high-affinity Ca indicator Oregon Green BAPTA 488-1 (200 $\mu \mathrm{M})$. The advantage of this indicator is that it permitted the visualization of $\mathrm{Ca}$ signals evoked by synaptic as well as by rebound stimuli. The disadvantage is that the high affinity of the indicator is expected to prolong and spread the measured Ca signals. Bearing in mind the limitations imposed by the dye, we reasoned that we might nevertheless be able to detect salient phenomena such as changes in baseline $\mathrm{Ca}$ with spiking, or the interaction of synaptic and rebound $\mathrm{Ca}$ currents to generate Ca spikes.

To elicit rebound Ca signals, cells were voltage-clamped at $-65 \mathrm{mV}$, stepped to voltages from $-75 \mathrm{mV}$ to $-115 \mathrm{mV}$ for 300 $\mathrm{ms}$, and then depolarized to $-55 \mathrm{mV}$. These conditioning steps were used because they span the voltages over which T-type Ca channels recover from inactivation (Fox et al., 1987; McDonough and Bean, 1998; McRory et al., 2001). The step to $-55 \mathrm{mV}$ evoked an inward current, probably dominated by low-voltage-activated T-type and possibly R-type Ca currents (Fig. 3A, top, black). Simultaneous monitoring of Ca signals in the soma and dendrite indicated that, on depolarization to $-55 \mathrm{mV}$ from $-115 \mathrm{mV}$, the Ca signal increased modestly at the soma $(4.6 \pm 1.1 \%, N=13)$ and to a larger extent in the dendrites $20-50 \mu \mathrm{m}$ from soma $(17.9 \pm 3.4 \%, N=13$ ) (Fig. $3 A$, bottom, black). The rebound $\mathrm{Ca}$ increase was evident throughout the dendrite and tended to become larger at progressively more distal locations (Fig. 3D), consistent with previous reports that low-voltage-activated Ca channels are present at higher densities in the dendrites of these neurons (Muri and Knöpfel, 1994; Gauck et al., 2001). The peak amplitude of current recorded in the soma after the step back to $-55 \mathrm{mV}$ was not strongly correlated with the peak amplitude of the Ca signal recorded in the dendrite $\left(R^{2}=0.14, N=13\right)$ (supplemental Fig. 1, available at www.jneurosci.org as supplemental material). This lack of correlation might arise if small currents flowed into small compartments, producing large local Ca changes, or if the voltage-gated Ca influx induced release from Ca stores. Alternatively, in some cases the imaging sites might have been electrically remote, although this possibility seems less likely, given that these sites were relatively close to the soma $(<100 \mu \mathrm{m})$.

To test whether voltage-clamp protocols that eliminate (somatic) Ca current were effective at reducing the rebound $\mathrm{Ca}$ signal, we repeated the experiments but with voltage ramps after the step hyperpolarizations. Indeed, the peak dendritic signal during a $400 \mathrm{~ms}$ ramp to $-55 \mathrm{mV}$ was reduced by $72 \pm 8 \%$ relative to that produced by the step $(N=4)$ (Fig. $3 A$, gray), suggesting that the dendritic recording sites were reasonably voltage-clamped. Moreover, because our previous work showed that voltage ramps applied after hyperpolarizations block the induction of plasticity (Pugh and Raman, 2006), these data support the idea that a necessary consequence of the hyperpolarization during effective potentiation protocols is a $\mathrm{Ca}$ increase resulting from synchronous activation of voltage-gated Ca channels after hyperpolarization is rapidly relieved.

Next, to measure synaptic signals, neurons were voltageclamped at $-65 \mathrm{mV}$. Previous studies indicate that NMDA receptors of cerebellar nuclear neurons pass current in standard ACSF even at these negative voltages, owing to a weak $\mathrm{Mg}^{2+}$ block (Audinat et al., 1992; Anchisi et al., 2001; Pugh and Raman, 2006). Therefore, to identify sites of putative synaptic Ca influx, 
we imaged a length of dendrite while applying a $100 \mathrm{~Hz}$ train of stimuli to excitatory afferents. Scanning the dendrite revealed relatively small ( $\sim 2-3 \mu \mathrm{m}$ diameter $)$ dendritic regions in which synaptically evoked Ca transients could be observed, with little or no change in fluorescence in adjacent sites (Fig. 3C,D), suggesting that these regions included activated synapses. Synaptically evoked relative $\mathrm{Ca}$ changes within these putative synaptic sites ranged from 4 to $23 \%$ with a mean change of $12.9 \pm 1.6 \%(N=$ 15 synapses on 12 cells).

\section{Testing a threshold rule for potentiation}

Having verified that we could detect synaptic and rebound $\mathrm{Ca}$ signals, we next evaluated the threshold rule by testing whether induction protocols that are effective, i.e., that reliably and robustly induce potentiation, evoke a Ca rise that is larger than that produced by ineffective protocols at the synapse. In these experiments, we identified putative synaptic sites as in Figure 3, and then recorded $\mathrm{Ca}$ signals at these sites against a background of spontaneous firing. We first elicited synaptic and rebound $\mathrm{Ca}$ signals in isolation (Fig. $4 A$, top two traces, $B$ ), and then the two stimuli were applied together to generate either an effective potentiation protocol, with the synaptic stimulus beginning before the rebound, or an ineffective potentiation protocol, with the synaptic stimulus coinciding with the rebound (Fig. $4 \mathrm{~A}$, bottom two traces, $B$ ). In all protocols, the effect of synaptic stimulation was either to increase the firing rate or to drive cells into depolarization block, both of which raised $\mathrm{Ca}$ above the spontaneously firing baseline level. Conversely, hyperpolarization interrupted firing, leading to a decrease in dendritic $\mathrm{Ca}$. On resumption of firing, Ca rose again, to a level that sometimes but not always exceeded the baseline level.

To quantify these data, the maximal Ca change from the level maintained during spontaneous firing was measured during three time windows: the $250 \mathrm{~ms}$ preceding the hyperpolarization, the $150 \mathrm{~ms}$ hyperpolarization, and the $800 \mathrm{~ms}$ after the end of the hyperpolarization. The mean data are plotted in Figure $4 \mathrm{C}$. Notably, the Ca levels attained by the effective (synaptic preceding rebound) induction protocol are intermediate to those achieved with the other protocols, which are ineffective at potentiating EPSCs. With the imaging techniques used, however, the Ca signals in both current clamp and voltage clamp are likely to be highly filtered, so that local transients were either prevented or not detected, and the mobile dye is expected to extend the spatial range of the signals. Nevertheless, it is evident that the combination of synaptic and rebound stimulation does not produce an overtly supralinear $\mathrm{Ca}$ increase, suggesting that regenerative $\mathrm{Ca}$ spikes are not triggered by the combined stimuli, unlike at other excitatory cerebellar synapses (Brenowitz and Regehr, 2005). Moreover, because synaptic stimulation alone generates the largest, longest $\mathrm{Ca}$ increase, these data provide evidence against a threshold rule. They also do not support the inverse-threshold rule, because the ineffective (rebound preceding synaptic) induction protocol produces a smaller Ca rise.

This interpretation is subject to two concerns, however. First, potentiation was not measured in these experiments, because the time required to locate putative synaptic sites and then to induce and measure plasticity exceeded the possible recording durations. Therefore, it is possible that binding of $\mathrm{Ca}$ by the indicator might have prevented release from $\mathrm{Ca}$ stores or interfered with activation of Ca-dependent enzymes, so that the Ca signals recorded corresponded to levels that would not have been able to induce plasticity. We therefore tested whether potentiation could be induced in the presence of the indicator (without concurrent
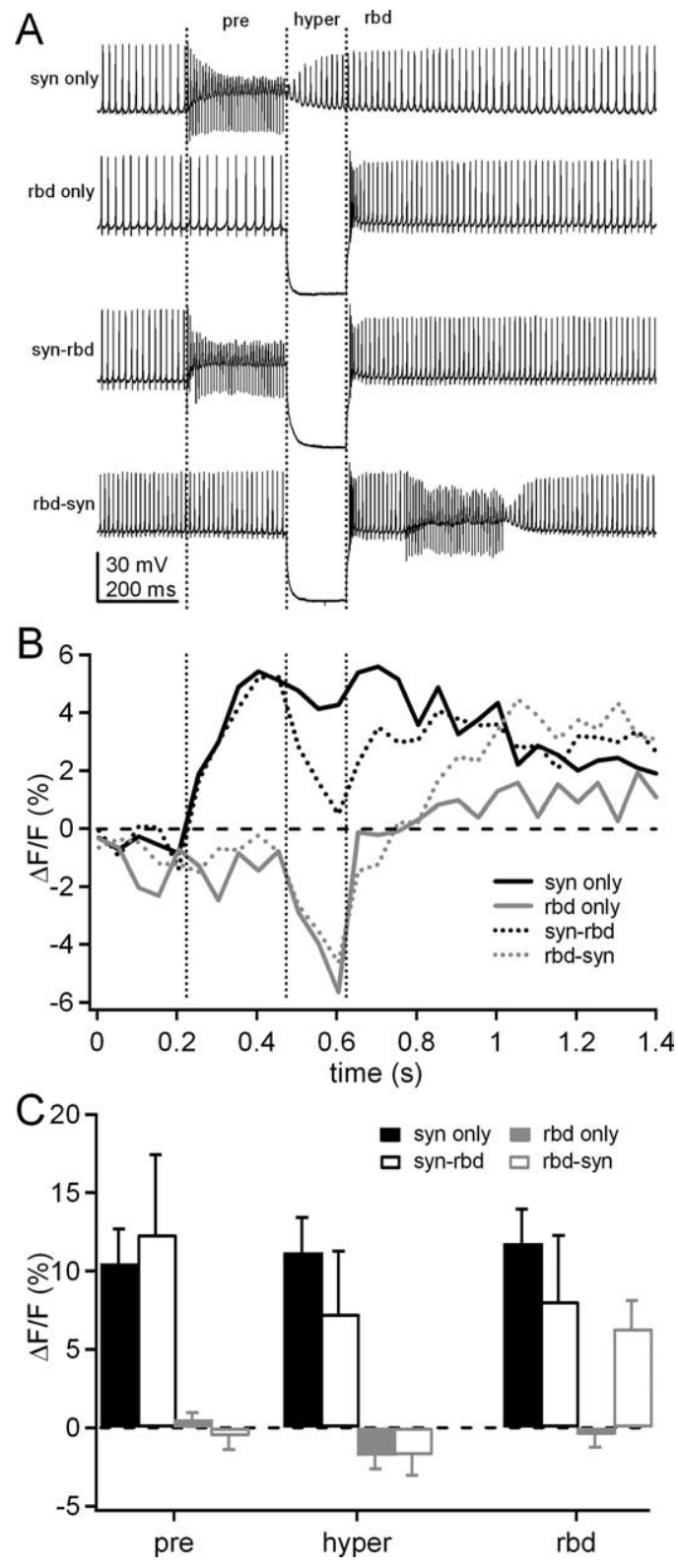

Figure 4. $\boldsymbol{A}-\boldsymbol{C}$, Ca signals elicited in nuclear cell dendrites by effective and ineffective current-clamp induction protocols. Representative voltage traces $(\boldsymbol{A})$ and Ca signals $(\boldsymbol{B})$ evoked at putative synaptic sites by synaptic stimulation applied alone (syn only), hyperpolarization and rebound response alone (rbd only), the synaptic train $400 \mathrm{~ms}$ preceding the rebound response (syn-rbd), and the synaptic train $150 \mathrm{~ms}$ after the rebound response (rbd-syn). Changes are measured relative to the mean Ca level maintained during spontaneous firing. Vertical lines indicate the onset of synaptic and rebound responses in the syn-rbd graph and define windows for measurements in $\boldsymbol{C}$. $\boldsymbol{C}$, Mean maximal Ca changes (increases or decreases) from the four conditions in $\boldsymbol{A}$, measured during the period preceding the hyperpolarization (pre), during the hyperpolarization (hyper), and during rebound ( $r b d)$.

imaging). Indeed, with $200 \mu \mathrm{M}$ Oregon Green BAPTA 488-1 in the pipette, the effective protocol of Figure 4 still potentiated EPSCs by $20.3 \pm 7.5 \%(N=5)$ (data contributed by Dr. A. L. Person, laboratory of I.M.R.). These results rule out the possibility that the Ca signals measured in the presence of indicator correspond to protocols that could not have induced potentiation.

The second concern results from the fact that highly localized $\mathrm{Ca}$ transients were beyond the resolution of our recording techniques. It therefore remains possible that the synaptic and rebound stimuli in the effective protocol may have either produced 



Figure 5. Ca signals elicited in nuclear cell dendrites by effective and ineffective voltageclamp induction protocols. $\boldsymbol{A}, \boldsymbol{B}$, Representative currents $(\boldsymbol{A})$ and Ca signals $(\boldsymbol{B})$ evoked at putative synaptic sites by synaptic stimulation applied alone (syn only), hyperpolarization and rebound current alone (rbd only), the onset of the synaptic train 350 ms preceding the rebound current (syn-rbd), and the onset of the EPS (train coinciding with the rebound current (rbd-syn). Vertical lines indicate the onset of synaptic and rebound currents in the syn-rbd graph. C, Mean Ca signals averaged $>350 \mathrm{~ms}$ beginning at the time of the peak rebound current. Striped bar indicates the sum of the signals evoked by synaptic and rebound stimuli applied alone. Asterisk indicates statistically significant difference; NS, nonsignificant difference.

a particularly high level of Ca or generated the precise local Ca concentrations necessary to induce potentiation. To explore these possibilities, we altered the background Ca level by repeating recordings in voltage-clamped neurons. The suppression of spikes allowed Ca changes from the synaptic and rebound stimuli to be recorded in isolation. Under voltage clamp, the baseline levels of $\mathrm{Ca}$ at putative synaptic regions were reduced by $18.4 \pm$ $4.8 \%(N=7)$, relative to when cells were spontaneously firing. We again recorded $\mathrm{Ca}$ signals resulting from either synaptic or rebound stimulation, as well as the Ca signals obtained when the stimuli were combined in the temporal sequence that either favors or fails to induce potentiation (Fig. $5 A, B$ ). For each condition, the Ca signal was measured for $350 \mathrm{~ms}$ after the peak of the rebound current. Within this time window, synaptic or rebound stimulation applied alone elicited relative $\mathrm{Ca}$ changes of $4.4 \pm$ $0.9 \%$ or $6.8 \pm 1 \%$, respectively (Fig. $5 C$ ). When the stimuli were applied concurrently in the effective protocol, however, the total Ca signal was not significantly different from the linear sum of its components $(10.4 \pm 1.6 \%$ vs $11.2 \pm 1.6 \%, p=0.52, N=8$ synapses from 6 cells) (Fig. $5 C$ ), again suggesting that no additional Ca sources were recruited by the combined stimuli. Moreover, in the ineffective protocol, the combined stimuli consistently produced a larger peak Ca signal than in the effective protocol ( $14.9 \pm 2.9 \%$ vs $10.4 \pm 1.6 \%, N=5, p=0.02$ ) (Fig. $5 C$ ). Thus, although the absolute levels of Ca were greatly reduced in voltage clamp because action potentials were suppressed, the Ca changes produced by the effective induction protocol were once again bracketed by those generated by ineffective protocols. Even considering the fact that the high-affinity $\mathrm{Ca}$ indicator would have extended the apparent duration of the synaptic and rebound signals, it is unlikely that the effective current-clamp and voltageclamp protocols produced an identical profile of Ca concentrations, providing further evidence against either a threshold or inverse-threshold rule.

\section{Input specificity of potentiation}

The results are instead more consistent with the "priming rule" mentioned above, in which the synaptic and hyperpolarization/ rebound Ca signals act independently to activate distinct intracellular signaling pathways, both (or all) of which are required to induce potentiation. Because this hypothesis requires that the hyperpolarization/rebound triggers potentiation exclusively at previously primed synapses, however, it makes the specific prediction that potentiation must be limited to synapses activated during the induction protocol.

We therefore tested the input specificity of potentiation by recording EPSCs elicited by two independent sets of excitatory synapses onto a nuclear neuron and delivering the induction protocol to only one set of synapses (the "test pathway"). To maximize the probability of activating independent populations of synapses, two stimulating electrodes were inserted far apart in the white matter surrounding the cerebellar nuclei, with one at the ventral surface of the cerebellum and one in the corticonuclear tract. The stimulus intensity was adjusted to produce EPSCs of similar amplitudes in the two pathways. Because they could not always be made identical, sometimes the larger and sometimes the smaller EPSC was selected as the test pathway. Across cells, however, the mean amplitudes of the initial EPSCs in the control and test pathways were nearly the same $(256 \pm 42 \mathrm{pA}$ and $255 \pm$ $34 \mathrm{pA}, N=6$ ). Because these EPSCs depressed only mildly in response to paired-pulse stimulation, an insensitivity of one pathway to previous stimulation of the other pathway did not provide a rigorous test of pathway independence. Instead, independence was tested by evoking EPSCs in the two pathways, first separately and then simultaneously, and then comparing the EPSC evoked by simultaneous stimulation with the sum of the separately evoked EPSCs. Only cells in which the amplitude and kinetics of the summed EPSC overlapped with the EPSC produced by simultaneous stimulation were tested further for potentiation (Fig. 6A).

After establishing a baseline amplitude of EPSCs for each set of synapses, the effective induction protocol of Figure $1 A$ was applied in current clamp to the test pathway, whereas the other "control pathway" was not activated. After induction, EPSCs in the test pathway increased by $57 \pm 20 \%(p=0.06, N=6)$, whereas those in the control pathway remained unchanged 



Figure 6. Synapse specificity of potentiation. $A$, EPSCs evoked by stimulation of the control pathway (thin gray trace), the test pathway (thick black trace), or both simultaneously (thick gray trace). Thin black trace, the sum of control and test EPSCS, confirming independence by overlap with the thick gray trace. $\boldsymbol{B}$, Mean EPSCs from the control and test pathways before (thick traces) and after (thin traces) induction protocols in which synaptic stimuli were applied only to the test pathway. EPSCs are normalized to the preinduction amplitude and superimposed for comparison. C, Mean change in EPSC amplitudes in response to induction in the two pathways.

$(-4 \pm 4 \%, p=0.6)$ (Fig. $6 B, C)$. Thus, potentiation is indeed limited to synapses activated by the induction protocol, satisfying the basic requirement of a priming rule, as well as suggesting that the synaptic signal is localized. The results also verify that the rebound stimulus alone is inadequate to potentiate synapses (Pugh and Raman, 2006), instead supporting the idea that the widespread postinhibitory Ca signal is only an effective trigger of potentiation at recently activated synapses.

\section{Inducing plasticity with synaptic inhibition instead of current injections}

In all induction protocols tested, we have mimicked postsynaptic inhibition with current injections that hyperpolarize neurons to voltages between -80 and $-90 \mathrm{mV}$. To a first approximation, this stimulus provides a reasonable simulation of inhibition, because it precisely dictates the times when firing ceases and when it resumes. Nevertheless, current injections and Purkinje-mediated IPSPs differ in the extent of hyperpolarization produced. In cerebellar nuclear neurons, the chloride equilibrium potential is
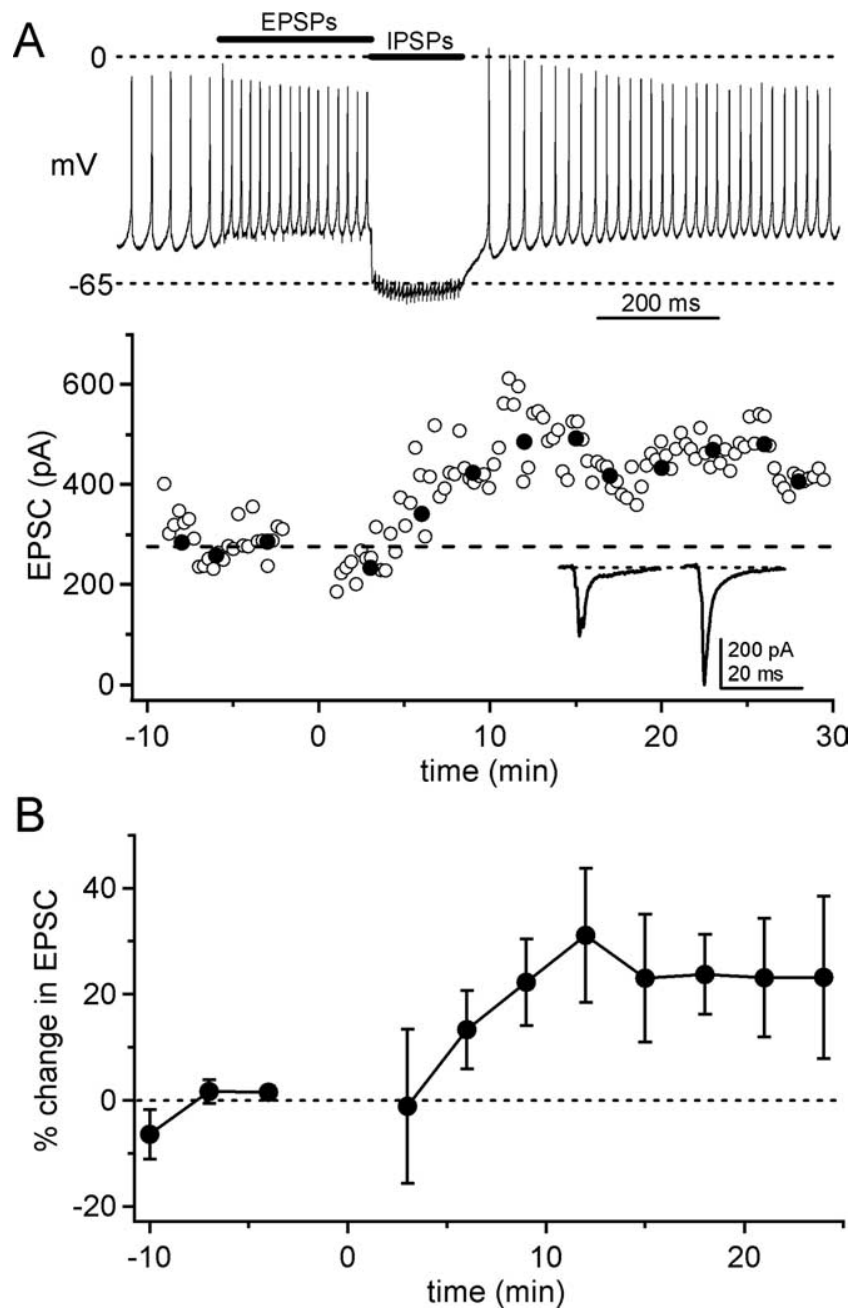

Figure 7. Potentiation in response to synaptic excitation and synaptic inhibition. $\boldsymbol{A}$, Top, Representative response of a spontaneously firing nuclear neuron to the effective induction protocol of Figure $2 A$, but with hyperpolarization replaced by $100 \mathrm{~Hz}$ stimulation of mixed IPSPS and EPSPS, with IPSPs predominating. Bottom, EPSC amplitudes before and after stimulation for the cell shown above. Open circles are individual EPSCs; solid circles are the mean of the 10 surrounding EPSCs. Inset, Mean of 10 EPSCs in this cell before and after induction. $\boldsymbol{B}$, Mean change in EPSC amplitude before and after stimulation.

near $-75 \mathrm{mV}$ (Jahnsen, 1986b; Aizenman and Linden, 1999), and the membrane potential during trains of IPSPs falls just short of this value (Telgkamp and Raman, 2002). Therefore, physiological inhibition is likely to recruit smaller rebound responses than do steps to more negative voltages, raising the question of the efficacy of "real" IPSPs at providing a triggering signal.

We therefore tested whether potentiation of EPSCs could be induced under more physiological conditions, i.e., with Purkinjemediated inhibition evoking postinhibitory firing. In these experiments, SR95531 was omitted from the bath to permit activation of $\mathrm{GABA}_{\mathrm{A}}$ receptors. Two stimulating electrodes were inserted into the white matter surrounding the cerebellar nuclei (see Materials and Methods). One electrode was positioned to evoke exclusively excitatory responses (in the "test" pathway). The other electrode was placed deep in the corticonuclear tract, where it elicited IPSPs as well as EPSPs (the "mixed PSP pathway"), with IPSPs predominating, resulting in a net inhibition of firing in the nuclear neuron (Fig. 7A, top). We substituted stimulation of this pathway for the hyperpolarizing current injection in the effective induction protocol of Figure $1 A$ and measured the 
effect on EPSCs in the test pathway. To mimic physiological conditions as closely as possible, we again allowed the cell to fire spontaneously in the intervals between applications of the induction protocol. With this induction protocol, EPSCs in the test pathway were potentiated by $30 \pm 10 \%(N=6, p=0.06 ; 66 \%$ reliability) (Fig. 7A, bottom, $B$ ). Thus, under nearly physiological conditions that include background spontaneous firing and Purkinje-mediated inhibition, sequential synaptic excitation and inhibition does indeed potentiate EPSCs. Interestingly, the mixed PSPs hyperpolarized the membrane potential to only $-67 \pm 1$ $\mathrm{mV}$ (Fig. 7A). Because this voltage is not negative enough for extensive recovery of most low-voltage-activated $\mathrm{Ca}$ channels (Fox et al., 1987; McDonough and Bean, 1998; McRory et al., 2001), it suggests that the majority of postinhibitory rebound Ca flows through high-voltage-activated $\mathrm{Ca}$ channels activated by the resumption of firing. Moreover, because the interruption of spontaneous firing reduces basal Ca levels (Fig. 4), these data raise the possibility that the triggering signal may involve a decrease in intracellular Ca while the cell is silenced, as well as an increase in $\mathrm{Ca}$ after the inhibition.

\section{Potentiation requires both kinase and phosphatase activity}

Together, these observations support the idea that the synaptic and inhibition/rebound stimuli activate distinct intracellular signaling pathways. Because both stimuli affect postsynaptic Ca levels, it is likely that these signaling pathways are sensitive to changes in local Ca concentration. We therefore tested whether Ca-dependent enzymes that are involved in several forms of potentiation and/or depression might also be required for plasticity of EPSCs in the cerebellar nuclei. These included CaMKII (Malinow et al., 1988; Silva et al., 1992a,b), calcineurin (Mulkey et al., 1993, 1994; Belmeguenai and Hansel, 2005), and PKC (Chung et al., 2000; Seidenman et al., 2003). We first blocked CaMKII activity by including a CaMKII-inhibitory peptide (fragment 290 $309,25 \mu \mathrm{M}$ ) in the intracellular solution and repeating the most effective induction protocol of Figure 1 (with the synaptic stimulus $400 \mathrm{~ms}$ before the rebound). After the protocol, EPSCs initially increased in amplitude with a time course similar to that of potentiation under control conditions. Nevertheless, EPSCs consistently returned to the baseline amplitude within $\sim 20 \mathrm{~min}$ (Fig. $8 A)(N=7)$. To corroborate this result, we repeated the experiment with the membrane-permeant CaMKII inhibitor KN62 included in the bath solution. Under these conditions, EPSCs returned to baseline after induction $(N=3)$. Pooling the data from both drugs indicates that when CaMKII was blocked, induction protocols produced no significant long-lasting change in EPSC amplitudes $(8.6 \pm 4.1 \%, N=10)$. The mean pooled data are shown in Figure $8 B$.

When the activation of calcineurin (protein phosphatase 2B) was blocked by either $50 \mu \mathrm{M}$ intracellular calcineurinautoinhibitory peptide $(N=6)$ or $1 \mu \mathrm{M}$ extracellular FK-506 $(N=5)$, EPSCs did not potentiate at all and instead depressed by $12 \pm 7 \%(p<0.01$ Fig. $8 A, B)$. Thus, not only is an increase in calcineurin activity required for the induction of potentiation, but the same induction protocols can also elicit a long-lasting depression when this enzyme is suppressed. These results are consistent with the idea that multiple processes can be triggered by the induction protocols and raise the possibility that the slow onset of potentiation may reflect the time course of calcineurin action. Moreover, these data illustrate that activity by both kinase and phosphatase is required for stable potentiation, suggesting that multiple substrates must be modulated for this form of plasticity to occur. In contrast, when PKC activity was reduced by
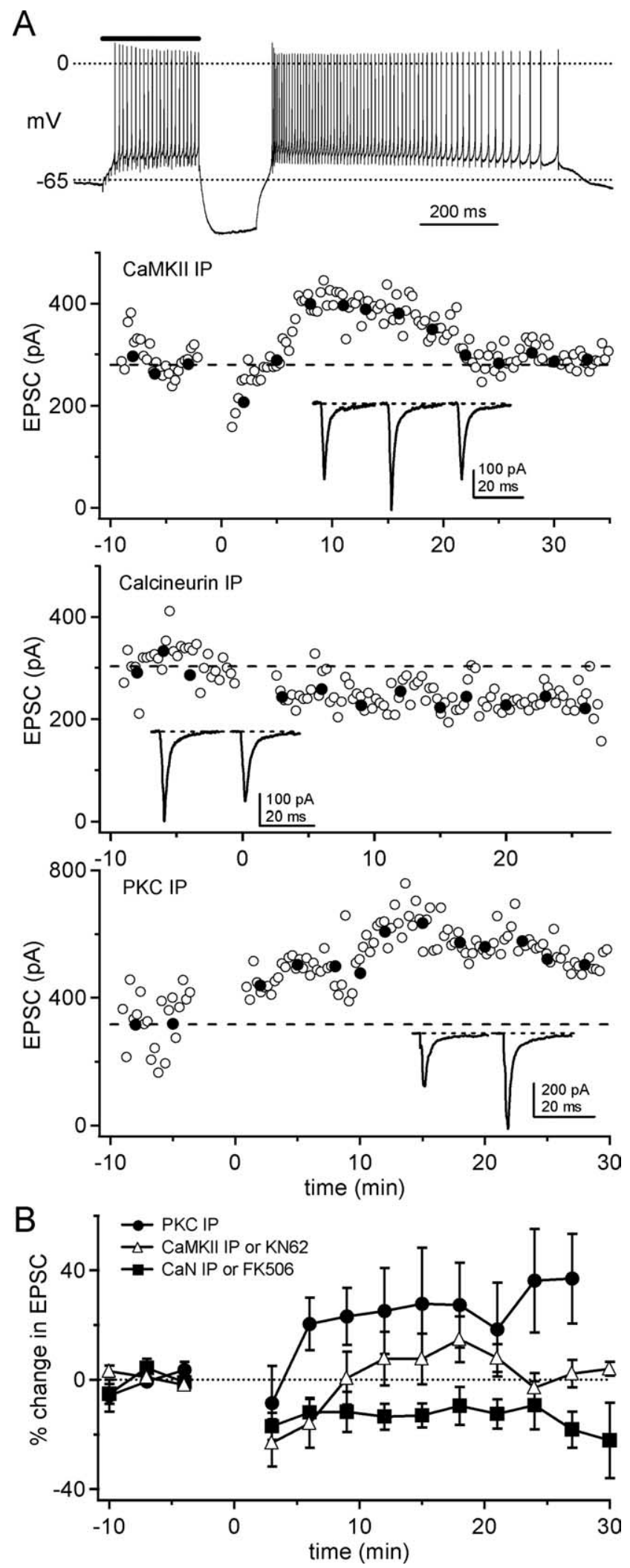

Figure 8. Requirement for active CaMKII and calcineurin, but not PKC, in potentiation. $\boldsymbol{A}$, Top, Representative response to the effective induction protocol of Figure $1 A$ in a cell containing calcineurin autoinhibitory peptide. Bottom, EPSC amplitudes in three cells before and after induction in the presence of inhibitory peptides (IP) blocking activity of CaMKII, calcineurin, or PKC (as labeled). Insets, Mean of 10 EPSCs before and after induction for each condition. For inhibition of CaMKIl, three EPSCS are shown. The first is preinduction, the second $10 \mathrm{~min}$ postinduction, and the third 24 min postinduction. $\boldsymbol{B}$, Mean changes in EPSC amplitudes during inhibition of CaMKII (triangles), calcineurin (squares), and PKC (circles). 
including the inhibitory peptide $\mathrm{PKC}_{19-31}(1-2 \mu \mathrm{M})$ in the intracellular solution, potentiation was unchanged relative to control ( $p=0.32$, unpaired $t$ test, $N=6$ ) (Fig. $8 A, B$, circles). The effects of these drugs on plasticity did not appear to result from direct modification of either synaptic currents or intrinsic excitability. Specifically, none of the drugs changed EPSC kinetics relative to control, with the exception of KN62, which slightly slowed the fast decay constant of the EPSC. Additionally, the number of action potentials produced in the first $300 \mathrm{~ms}$ of the rebound depolarization was not significantly changed in any of the inhibitors (supplemental Fig. 2, available at www.jneurosci.org as supplemental material). Together, these data suggest that neither priming nor triggering depends strongly on the activation of PKC. In contrast, both CaMKII and calcineurin are required for long-term plasticity.

\section{Discussion}

These experiments provide evidence that excitation and inhibition interact to potentiate mossy fiber EPSCs in cerebellar nuclear neurons. This interaction occurs when an $\sim 250 \mathrm{~ms}$ train of synaptic excitation precedes the offset of a $150 \mathrm{~ms}$ period of inhibition by $\sim 400 \mathrm{~ms}$. Consequently, potentiation requires an approximate coincidence of synaptic excitation and inhibition, a pattern of activity that likely occurs during cerebellar learning. The excitatory synaptic and postinhibitory rebound stimuli activate NMDA receptors and voltage-gated channels, respectively, each of which elevates $\mathrm{Ca}$ in the dendritic shaft. The sign of plasticity is unaffected by raising or lowering basal dendritic $\mathrm{Ca}$, providing evidence against the idea that potentiation proceeds when Ca levels reach a threshold. Instead, excitatory and inhibitory stimuli apparently produce functionally distinct local (priming) and global (triggering) signals that induce plasticity through a multistep process, involving the Ca-dependent enzymes calcineurin and CaMKII.

\section{Plasticity rules in the cerebellar nuclei}

These rules for inducing potentiation are distinct from classical long-term potentiation (LTP) in the hippocampal CA1 region, in which EPSCs are potentiated by large increases in postsynaptic Ca resulting from a temporal coincidence, on a scale of a few milliseconds, of synaptic excitation and postsynaptic spiking (Markram et al., 1997; Bi and Poo, 1998). The converse occurs at parallel fiber synapses onto Purkinje neurons, where small elevations of $\mathrm{Ca}$ promote potentiation (Coesmans et al., 2004; Belmeguenai and Hansel, 2005; Jörntell and Hansel, 2006). In cerebellar nuclear neurons, at least two factors may make such threshold or inverse-threshold rules inappropriate. First, spontaneous firing raises $\mathrm{Ca}$ levels in the relatively short dendrites of nuclear cells, near excitatory synapses. Thus, Ca-dependent induction of plasticity of EPSCs must occur against a high, probably fluctuating, background Ca level, which is expected to add noise to a threshold-based induction signal. Second, behaviorally relevant information from the external world likely increases inhibition from Purkinje cells as well as excitation from mossy fibers. Because inhibition decreases $\mathrm{Ca}$, and excitation increases $\mathrm{Ca}$, a simple threshold rule may be inadequate to encode meaningful temporal information that is required for adaptive plasticity.

The alternative hypothesis, supported by our data, is that excitatory inputs generate Ca signals that prime synapses for potentiation, whereas inhibitory inputs trigger potentiation by first decreasing and then increasing free $\mathrm{Ca}$. Because these events must occur serially, the timing of synaptic input relative to the rebound determines whether potentiation occurs. Several studies provide precedent for the idea that the temporal sequence of activation of different Ca sources sets the sign of plasticity. For instance, in pyramidal neurons of rat somatosensory cortex, synaptic activity preceding spiking induces LTP via NMDA receptor activation, whereas the reverse sequence induces LTD, because voltagegated $\mathrm{Ca}$ influx facilitates metabotropic glutamate receptormediated endocannabinoid release (Bender et al., 2006; Nevian and Sakmann, 2006). In the dorsal cochlear nucleus, synaptic and spike-mediated Ca influx activates CaMKII, promoting LTP, and endocannabinoid release, promoting LTD. The former dominates when spiking precedes synaptic stimuli, however; the latter occurs when the order is reversed (Tzounopoulos et al., 2007).

In the case of the cerebellar nuclei, because potentiation requires NMDA receptor activation (Pugh and Raman, 2006), priming of synapses is probably initiated by $\mathrm{Ca}$ influx through these receptors, although other Ca-permeable glutamate receptors may contribute as well. Because of a weak $\mathrm{Mg}^{2+}$ block of NMDA receptors in nuclear cells (Anchisi et al., 2001; Pugh and Raman, 2006), the synaptic Ca signal is probably minimally affected by postsynaptic spiking. Although most excitatory synapses are located on dendritic shafts, this signal must be highly localized, possibly by endogenous buffering, as in aspiny cerebellar stellate neurons (Soler-Llavina and Sabatini, 2006). Moreover, at other excitatory synapses, calcineurin is localized to the postsynaptic density via A-kinase anchoring proteins (Coghlan et al., 1995; Gomez et al., 2002) and is stimulated by activation of NMDA receptors (Mulkey et al., 1993, 1994; Tong et al., 1995). The biochemical association of NMDA receptors and calcineurin, along with the result that inhibition of calcineurin consistently prevented potentiation, makes it reasonable to hypothesize that activation of the phosphatase by NMDA receptormediated Ca influx is a key step in the priming of mossy fiber synapses onto nuclear cells.

The triggering signal, in contrast, appears to be a global signal initiated by inhibition. Triggering may depend on the Ca increase after a period of hyperpolarization via the activation of Ca currents (Llinás and Mühlethaler, 1988; Aizenman and Linden, 1999; Molineux et al., 2006), and/or by the Ca decrease from the silencing of spontaneous firing by inhibition. Our previous work indicated that low-voltage-activated rebound currents were necessary to potentiate EPSCs in nuclear cells that were prevented from firing by voltage clamp (Pugh and Raman, 2006), suggesting that triggering requires a postinhibitory increase in $\mathrm{Ca}$. This result does not, however, rule out the possibility that the interruption of firing itself, and the concomitant decrease in $\mathrm{Ca}$, also contributes to the triggering signal in normally firing cells. Studies of medial vestibular nuclear neurons, which are also Purkinje cell targets, provide precedent for this idea. In these cells, inhibiting spontaneous firing decreases intracellular $\mathrm{Ca}$, reducing the activity of tonically active CaMKII and producing long-term changes in intrinsic excitability (Nelson et al., 2003, 2005). Similarly, in cerebellar nuclear neurons, the basal level of Ca shifts significantly depending on whether or not the cell is firing, raising the possibility that triggering comprises the inhibition of $\mathrm{Ca}$ dependent processes followed by the activation (or resumption) of other Ca-dependent processes.

This scenario is appealing in part because trains of IPSPs actually hyperpolarize cells quite modestly, only to -65 to $-70 \mathrm{mV}$ (Telgkamp and Raman, 2002; McKay et al., 2005). This voltage range is unlikely to produce significant recovery of low-voltageactivated Ca currents (McDonough and Bean, 1998; McRory et al., 2001), but potentiation proceeds nonetheless. Therefore, although low-voltage-activated Ca currents have the capacity to carry the triggering signal in voltage-clamped cells, it seems prob- 
able that the postinhibitory Ca signal under physiological conditions instead arises from high-voltage-activated $\mathrm{Ca}$ currents activated during spiking, which either transiently raise Ca or simply restore it to baseline levels.

\section{Molecular bases for priming and triggering}

Although the stability of the paired-pulse ratio as well as the EPSC variance indicates a postsynaptic site of plasticity, the molecular events underlying priming and triggering are unknown. Interestingly, potentiation was more reliable and robust when induction protocols were applied against a background of spontaneous firing, suggesting that synaptic excitation is most effective when $\mathrm{Ca}$ levels start high. Mechanistically, these observations suggest that triggering may involve a shift in the balance of two antagonistic processes such as receptor internalization and receptor insertion. If these processes depend on separate signaling pathways with distinct Ca sensitivities (Chung et al., 2000; Hayashi et al., 2000; Seidenman et al., 2003), a drop followed by an increase in $\mathrm{Ca}$ might favor receptor insertion at previously primed synapses. Such a situation could arise if phosphatases or kinases activated during priming can regulate PSD-95 (postsynaptic density protein 95) or TARPs (transmembrane AMPA-receptor regulatory proteins) to make a transiently permissive environment for AMPA receptor insertion (El-Husseini et al., 2002; Schnell et al., 2002; Tomita et al., 2005; Kim et al., 2007).

Alternatively, priming might recruit receptor pools to specific synapses. For instance, Esteban et al. (2003) proposed that PKAmediated phosphorylation readies GluR1 receptors for insertion, whereas CaMKII-mediated phosphorylation triggers fusion of GluR1-containing vesicles into synapses. A parallel mechanism may exist in nuclear neurons, although the receptors involved may differ, because in situ hybridizations reveal substantial GluR2, but little GluR1, in the cerebellar nuclei (Lein et al., 2007).

\section{Relating potentiation of EPSCs to cerebellar learning}

The form of plasticity described here has several characteristics appropriate for a cellular process relevant to delay eyelid conditioning. In this cerebellar task, conditioned stimuli are carried by mossy fibers, which likely provide nuclear cells with concurrent direct excitation and indirect inhibition, via the granule-Purkinje-nuclear cell circuit (Mauk et al., 1986; Steinmetz et al., 1989; Hesslow et al., 1999). Similarly, at the cellular level, cerebellar nuclear neurons detect the approximate coincidence of synaptic excitation and inhibition, in which a train of excitation precedes a postinhibitory rebound by $\sim 300 \mathrm{~ms}$. Acquisition of conditioned responses is optimal when conditioned stimuli last for 100-500 ms (Ohyama et al., 2003b), consistent with the durations of synaptic excitation that effectively potentiate EPSCs. A critical component of cellular plasticity is the relief of inhibition; early in delay eyelid conditioning training, such disinhibition of nuclear cells might occur after the unconditioned stimulus, which activates complex spikes in Purkinje cells. Complex spikes can be followed by pauses in Purkinje cell firing (Sato et al., 1992), which could permit postinhibitory firing in the nuclei. Later in training, Purkinje cells generally slow their firing toward the end of the conditioned stimulus, which would also disinhibit nuclear cells (Green and Steinmetz, 2005; Jirenhed et al., 2007). The latter scenario is consistent with the prediction that synaptic plasticity occurs first in the cerebellar cortex, and second in the nuclei (Medina and Mauk 1999). Finally, the synapse specificity of the potentiation of EPSCs in the cerebellar nuclei, coupled with a relief of Purkinje-mediated inhibition late in the conditioned stimulus, appears well suited to underlie the conditioned- stimulus specificity of delay eyelid conditioning (Ohyama et al., 2003a).

\section{References}

Aizenman CD, Linden DJ (1999) Regulation of the rebound depolarization and spontaneous firing patterns of deep nuclear neurons in slices of rat cerebellum. J Neurophysiol 82:1697-1709.

Aizenman CD, Linden DJ (2000) Rapid, synaptically driven increases in the intrinsic excitability of cerebellar deep nuclear neurons. Nat Neurosci 3:109-111.

Anchisi D, Scelfo B, Tempia F (2001) Postsynaptic currents in deep cerebellar nuclei. J Neurophysiol 85:323-331.

Audinat E, Gähwiler BH, Knöpfel T (1992) Excitatory synaptic potentials in neurons of the deep nuclei in olivo-cerebellar slice cultures. Neuroscience 49:903-911.

Belmeguenai A, Hansel C (2005) A role for protein phosphatases 1, 2A, and 2B in cerebellar long-term potentiation. J Neurosci 25:10768-10772.

Bender VA, Bender KJ, Brasier DJ, Feldman DE (2006) Two coincidence detectors for spike timing-dependent plasticity in somatosensory cortex. J Neurosci 26:4166-4177.

Bi GQ, Poo MM (1998) Synaptic modifications in cultured hippocampal neurons: dependence on spike timing, synaptic strength, and postsynaptic cell type. J Neurosci 18:10464-10472.

Brenowitz SD, Regehr WG (2005) Associative short-term synaptic plasticity mediated by endocannabinoids. Neuron 45:419-431.

Chan-Palay V (1977) Cerebellar dentate nucleus. Organization, cytology, and transmitters. Berlin: Springer.

Chung HJ, Xia J, Scannevin RH, Zhang X, Huganir RL (2000) Phosphorylation of the AMPA receptor subunit GluR2 differentially regulates its interaction with PDZ domain-containing proteins. J Neurosci 20:7258-7267

Coesmans M, Weber JT, De Zeeuw CI, Hansel C (2004) Bidirectional parallel fiber plasticity in the cerebellum under climbing fiber control. Neuron 44:691-700.

Coghlan VM, Perrino BA, Howard M, Langeberg LK, Hicks JB, Gallatin WM, Scott JD (1995) Association of protein kinase A and protein phosphatase 2B with a common anchoring protein. Science 267:108-111.

Egger V, Svoboda K, Mainen ZF (2005) Dendrodendritic synaptic signals in olfactory bulb granule cells: local spine boost and global low-threshold spike. J Neurosci 25:3521-3530.

El-Husseini Ael-D, Schnell E, Dakoji S, Sweeney N, Zhou Q, Prange O, Gauthier-Campbell C, Aguilera-Moreno A, Nicoll RA, Bredt DS (2002) Synaptic strength regulated by palmitate cycling on PSD-95. Cell 108:849-863.

Esteban JA, Shi SH, Wilson C, Nuriya M, Huganir RL, Malinow R (2003) PKA phosphorylation of AMPA receptor subunits controls synaptic trafficking underlying plasticity. Nat Neurosci 6:136-143.

Fox AP, Nowycky MC, Tsien RW (1987) Kinetic and pharmacological properties distinguishing three types of calcium currents in chick sensory neurones. J Physiol 394:149-172.

Frey U, Morris RG (1997) Synaptic tagging and long-term potentiation. Nature 385:533-536.

Garcia KS, Mauk MD (1998) Pharmacological analysis of cerebellar contributions to the timing and expression of conditioned eyelid responses. Neuropharmacology 37:471-480.

Gauck V, Thomann M, Jaeger D, Borst A (2001) Spatial distribution of lowand high voltage-activated calcium currents in neurons of the deep cerebellar nuclei. J Neurosci 21:RC158:1-4.

Gomez LL, Alam S, Smith KE, Horne E, Dell'Acqua ML (2002) Regulation of A-kinase anchoring protein 79/150-cAMP-dependent protein kinase postsynaptic targeting by NMDA receptor activation of calcineurin and remodeling of dendritic actin. J Neurosci 22:7027-7044.

Green JT, Steinmetz JE (2005) Purkinje cell activity in the cerebellar anterior lobe after rabbit eyeblink conditioning. Learn Mem 12:260-269.

Hayashi Y, Shi SH, Esteban JA, Piccini A, Poncer JC, Malinow R (2000) Driving AMPA receptors into synapses by LTP and CaMKII: requirement for GluR1 and PDZ domain interaction. Science 287:2262-2267.

Hesslow G (1994) Inhibition of classically conditioned eyeblink responses by stimulation of the cerebellar cortex in the decerebrate cat. J Physiol 476:245-256.

Hesslow G, Svensson P, Ivarsson M (1999) Learned movements elicited by 
direct stimulation of cerebellar mossy fiber afferents. Neuron 24:179-185.

Jahnsen H (1986a) Electrophysiological characteristics of neurones in the guinea-pig deep cerebellar nuclei in vitro. J Physiol 372:129-147.

Jahnsen H (1986b) Extracellular activation and membrane conductances of neurones in the guinea-pig deep cerebellar nuclei in vitro. J Physiol 372:149-168.

Jirenhed DA, Bengtsson F, Hesslow G (2007) Acquisition, extinction, and reacquisition of a cerebellar cortical memory trace. J Neurosci 27:2493-2502.

Jörntell H, Hansel C (2006) Synaptic memories upside down: bidirectional plasticity at cerebellar parallel fiber-Purkinje cell synapses. Neuron 52:227-238

Kim MJ, Futai K, Jo J, Hayashi Y, Cho K, Sheng M (2007) Synaptic accumulation of PSD-95 and synaptic function regulated by phosphorylation of serine-295 of PSD-95. Neuron 56:488-502.

Lein ES, Hawrylycz MJ, Ao N, Ayres M, Bensinger A, Bernard A, Boe AF, Boguski MS, Brockway KS, Byrnes EJ, Chen L, Chen L, Chen TM, Chin MC, Chong J, Crook BE, Czaplinska A, Dang CN, Datta S, Dee NR, et al. (2007) Genome-wide atlas of gene expression in the adult mouse brain. Nature 445:168-176.

Llinás R, Mühlethaler M (1988) Electrophysiology of guinea-pig cerebellar nuclear cells in the in vitro brain stem-cerebellar preparation. J Physiol 404:241-258.

Malinow R, Madison DV, Tsien RW (1988) Persistent protein kinase activity underlying long-term potentiation. Nature 335:820-824.

Markram H, Lübke J, Frotscher M, Sakmann B (1997) Regulation of synaptic efficacy by coincidence of postsynaptic APs and EPSPs. Science 275:213-215.

Martin KC, Casadio A, Zhu H, Yaping E, Rose JC, Chen M, Bailey CH, Kandel ER (1997) Synapse-specific, long-term facilitation of aplysia sensory to motor synapses: a function for local protein synthesis in memory storage. Cell 91:927-938.

Mauk MD, Steinmetz JE, Thompson RF (1986) Classical conditioning using stimulation of the inferior olive as the unconditioned stimulus. Proc Natl Acad Sci U S A 83:5349-5353.

McCormick DA, Thompson RF (1984) Cerebellum: essential involvement in the classically conditioned eyelid response. Science 223:296-299.

McDonough SI, Bean BP (1998) Mibefradil inhibition of T-type calcium channels in cerebellar Purkinje neurons. Mol Pharmacol 54:1080-1087.

McKay BE, Molineux ML, Mehaffey WH, Turner RW (2005) Kv1 K+ channels control Purkinje cell output to facilitate postsynaptic rebound discharge in deep cerebellar neurons. J Neurosci 25:1481-1492.

McRory JE, Santi CM, Hamming KS, Mezeyova J, Sutton KG, Baillie DL, Stea A, Snutch TP (2001) Molecular and functional characterization of a family of rat brain T-type calcium channels. J Biol Chem 276:3999-4011.

Medina JF, Mauk MD (1999) Simulations of cerebellar motor learning: computational analysis of plasticity at the mossy fiber to deep nucleus synapse. J Neurosci 19:7140-7151.

Miles FA, Lisberger SG (1981) Plasticity in the vestibulo-ocular reflex: a new hypothesis. Annu Rev Neurosci 4:273-299.

Molineux ML, McRory JE, McKay BE, Hamid J, Mehaffey WH, Rehak R, Snutch TP, Zamponi GW, Turner RW (2006) Specific T-type calcium channel isoforms are associated with distinct burst phenotypes in deep cerebellar nuclear neurons. Proc Natl Acad Sci U S A 103:5555-5560.

Morishita W, Sastry BR (1995) Pharmacological characterization of preand postsynaptic $\mathrm{GABA}_{\mathrm{B}}$ receptors in the deep nuclei of rat cerebellar slices. Neuroscience 68:1127-1137.

Mouginot D, Gähwiler BH (1995) Characterization of synaptic connections between cortex and deep nuclei of the rat cerebellum in vitro. Neuroscience 64:699-712.

Mulkey RM, Herron CE, Malenka RC (1993) An essential role for protein phosphatases in hippocampal long-term depression. Science 261:1051-1055.

Mulkey RM, Endo S, Shenolikar S, Malenka RC (1994) Involvement of a calcineurin/inhibitor-1 phosphatase cascade in hippocampal long-term depression. Nature 369:486-488.

Muri R, Knöpfel T (1994) Activity induced elevations of intracellular calcium concentration in neurons of the deep cerebellar nuclei. J Neurophysiol 71:420-428.

Nelson AB, Krispel CM, Sekirnjak C, du Lac S (2003) Long-lasting increases in intrinsic excitability triggered by inhibition. Neuron 40:609-620.
Nelson AB, Gittis AH, du Lac S (2005) Decreases in CaMKII activity trigger persistent potentiation of intrinsic excitability in spontaneously firing vestibular nucleus neurons. Neuron 46:623-631.

Nevian T, Sakmann B (2006) Spine Ca2+ signaling in spike-timingdependent plasticity. J Neurosci 26:11001-11013.

Ohyama T, Mauk M (2001) Latent acquisition of timed responses in cerebellar cortex. J Neurosci 21:682-690.

Ohyama T, Nores WL, Mauk MD (2003a) Stimulus generalization of conditioned eyelid responses produced without cerebellar cortex: implications for plasticity in the cerebellar nuclei. Learn Mem 10:346-354.

Ohyama T, Nores WL, Murphy M, Mauk MD (2003b) What the cerebellum computes. Trends Neurosci 26:222-227.

Ohyama T, Nores WL, Medina JF, Riusech FA, Mauk MD (2006) Learninginduced plasticity in deep cerebellar nucleus. J Neurosci 26:12656-12663.

Pugh JR, Raman IM (2005) $\mathrm{GABA}_{\mathrm{A}}$ receptor kinetics in the cerebellar nuclei: evidence for detection of transmitter from distant release sites. Biophys J 88:1740-1754

Pugh JR, Raman IM (2006) Potentiation of mossy fiber EPSCs in the cerebellar nuclei by NMDA receptor activation followed by postinhibitory rebound current. Neuron 51:113-123.

Raman IM, Gustafson AE, Padgett DE (2000) Ionic currents and spontaneous firing in neurons isolated from the cerebellar nuclei. J Neurosci 20:9004-9016.

Sato Y, Miura A, Fushiki H, Kawasaki T (1992) Short-term modulation of cerebellar Purkinje cell activity after spontaneous climbing fiber input. J Neurophysiol 68:2051-2062.

Schnell E, Sizemore M, Karimzadegan S, Chen L, Bredt DS, Nicoll RA (2002) Direct interactions between PSD-95 and stargazin control synaptic AMPA receptor number. Proc Natl Acad Sci U S A 99:13902-13907.

Seidenman KJ, Steinberg JP, Huganir R, Malinow R (2003) Glutamate receptor subunit 2 Serine 880 phosphorylation modulates synaptic transmission and mediates plasticity in CA1 pyramidal cells. J Neurosci 23:9220-9228.

Silva AJ, Stevens CF, Tonegawa S, Wang Y (1992a) Deficient hippocampal long-term potentiation in alpha-calcium-calmodulin kinase II mutant mice. Science 257:201-206.

Silva AJ, Paylor R, Wehner JM, Tonegawa S (1992b) Impaired spatial learning in alpha-calcium-calmodulin kinase II mutant mice. Science 257:206-211.

Soler-Llavina GJ, Sabatini BL (2006) Synapse-specific plasticity and compartmentalized signaling in cerebellar stellate cells. Nat Neurosci 9:798-806.

Steinmetz JE, Lavond DG, Thompson RF (1989) Classical conditioning in rabbits using pontine nucleus stimulation as a conditioned stimulus and inferior olive stimulation as an unconditioned stimulus. Synapse 3:225-233.

Telgkamp P, Raman IM (2002) Depression of inhibitory synaptic transmission between Purkinje cells and neurons of the cerebellar nuclei. J Neurosci 22:8447-8457.

Telgkamp P, Padgett DE, Ledoux VA, Woolley CS, Raman IM (2004) Maintenance of high-frequency inhibitory transmission at Purkinje to cerebellar nuclear synapses by spillover from boutons with multiple release sites. Neuron 41:113-126.

Thach WT (1968) Discharge of Purkinje and cerebellar nuclear neurons during rapidly alternating arm movements in the monkey. J Neurophysiol 31:785-797.

Tomita S, Stein V, Stocker TJ, Nicoll RA, Bredt DS (2005) Bidirectional synaptic plasticity regulated by phosphorylation of stargazin-like TARPs. Neuron 45:269-277.

Tong G, Shepherd D, Jahr CE (1995) Synaptic desensitization of NMDA receptors by calcineurin. Science 267:1510-1512.

Tzounopoulos T, Rubio ME, Keen JE, Trussell LO (2007) Coactivation of pre- and postsynaptic signaling mechanisms determines cellspecific spike-timing-dependent plasticity. Neuron 54:291-301.

Uusisaari M, Obata K, Knöpfel T (2007) Morphological and electrophysiological properties of GABAergic and non-GABAergic cells in the deep cerebellar nuclei. J Neurophysiol 97:901-911.

Wang SS, Denk W, Häusser M (2000) Coincidence detection in single dendritic spines mediated by calcium release. Nat Neurosci 3:1266-1273.

Zhang W, Linden DJ (2006) Long-term depression at the mossy fiber-deep cerebellar nucleus synapse. J Neurosci 26:6935-6944. 